\title{
Eccentric Figure-Eight Coils for Transcranial Magnetic Stimulation
}

\author{
Masaki Sekino $^{1,2^{*}}$, Hiroyuki Ohsaki ${ }^{1,3}$, Yoshihiro Takiyama ${ }^{1}$, Keita Yamamoto ${ }^{1}$, Taiga \\ Matsuzaki $^{2,4}$, Yoshihiro Yasumuro ${ }^{2,5}$, Atsushi Nishikawa ${ }^{2,6}$, Tomoyuki Maruo ${ }^{2}$, Koichi \\ Hosomi $^{2}$, and Youichi Saitoh ${ }^{2}$
}

${ }^{1}$ Department of Electrical Engineering and Information Systems, Graduate School of Engineering, the University of Tokyo, Tokyo, Japan

${ }^{2}$ Department of Neuromodulation and Neurosurgery, Graduate School of Medicine, Osaka University, Suita, Japan

${ }^{3}$ Department of Advanced Energy, Graduate School of Frontier Sciences, the University of Tokyo, Kashiwa, Japan

${ }^{4}$ Home Healthcare Research \& Development Planning Department, Teijin Pharma Limited

${ }^{5}$ Department of Civil, Environmental and Applied System Engineering, Faculty of Environmental Urban Engineering, Kansai University, Suita, Japan

${ }^{6}$ Bioengineering Course, Division of Mechanical Engineering and Robotics, Faculty of Textile Science and Technology, Shinshu University, Ueda, Japan

*Correspondence to: Masaki Sekino, 7-3-1 Hongo, Bunkyo-ku, Tokyo 113-8656, Japan. E-mail: sekino@bee.t.u-tokyo.ac.jp

Short running title: Eccentric Figure-Eight Coils for TMS 


\section{Grant Sponsors:}

This work was supported by the following three grants:

1. Strategic Research Program for Brain Sciences by the Ministry of Education, Culture, Sports, Science and Technology of Japan, and General Insurance Association of Japan

2. Program for Promotion of Fundamental Studies in Health Sciences of the National Institute of Biomedical Innovation.

3. Research fund from Teijin Pharma Limited.

Conflict of interest:

None 


\section{ABSTRACT}

Previously we proposed an eccentric figure-eight coil that can cause threshold stimulation in the brain at lower driving currents. In this study, we performed numerical simulations and magnetic stimulations to healthy subjects for evaluating the advantages of the eccentric coil. The simulations were performed using a simplified spherical brain model and a realistic human brain model. We found that the eccentric coil required a driving current intensity of approximately $18 \%$ less than that required by the concentric coil to cause comparable eddy current densities within the brain. The eddy current localization of the eccentric coil was slightly higher than that of the concentric coil. A prototype eccentric coil was designed and fabricated. Instead of winding a wire around a bobbin, we cut eccentric-spiral slits on the insulator cases, and a wire was woven through the slits. The coils were used to deliver magnetic stimulation to healthy subjects; among our results, we found that the current slew rate corresponding to motor threshold values for the concentric and eccentric coils were $86 \mathrm{~A} / \mu \mathrm{s}$ and $78 \mathrm{~A} / \mu \mathrm{s}$, respectively. The results indicate that the eccentric coil consistently requires a lower driving current to reach the motor threshold than does the concentric coil. Future development of compact magnetic stimulators will enable the treatment of some intractable neurological diseases at home.

Keywords: Transcranial magnetic stimulation; coil; brain; medical application; eddy current 


\section{INTRODUCTION}

Treatment of neuropathic pain such as central post-stroke pain is difficult in some cases in which patients are refractory to pharmacological therapy. For some drug-resistant patients, however, electrical stimulation can provide therapeutic effects through the use of implanted electrodes to continuously suppress their pain [Tsubokawa et al., 1991; Saitoh et al., 2000].

Our studies have shown that transcranial magnetic stimulation (TMS), in which eddy currents induced in the brain give rise to a therapeutic effect comparable to that of an electric stimulation, can be a useful alternative [Hirayama et al., 2006; Saitoh et al., 2007]. Because TMS provides magnetic stimulation through a coil located outside the patient's head, it has the advantage of being non-invasive. However, as the therapeutic effect of the TMS lasts for one day and decays with time, patients must undergo TMS daily to maintain pain relief.

In order to put the TMS therapy to better practical use, our goal is to develop a compact magnetic stimulator system suitable for installation in patients' homes [Kato et al., 2011; Okada et al., 2012; Yasumuro et al., 2012]. This system would be equipped with a coil navigation system to enable users to position the stimulator coil by themselves [Okada et al., 2012; Yasumuro et al., 2012].

As standard TMS achieves pain relief only when stimulations are applied repeatedly at 5 pulses per second or more [Saitoh et al., 2007], there is a limit to how much the driving circuits can be miniaturized, as delivery of repetitive stimulations requires heavy 
internal components such as capacitors and thyristors that can withstand high charging and discharging power. By requiring only single-shot stimulation, TMS systems could be substantially downsized [Epstein, 2008; De Sauvage et al., 2010].

In addition to weight, coil heating is a major technical challenge in repetitive TMS [Bischoff et al., 1994; Weyh et al., 2005], and although some commercialized TMS systems are equipped with water-cooled coils, they may cause maintenance problems when installed in a patient's home.

Coil design is one of the most important aspects of TMS and optimizing it could lead to reductions in the driving current and Joule heat. A variety of coil designs have previously been proposed and evaluated, including circular coils, which have been used since the first demonstration of TMS [Barker et al., 1985], and figure-eight coils, which consist of two circular windings in which currents flow in opposite directions [Ueno et al., 1988; Ueno et al., 1990]. The figure-eight design generates high eddy current densities under the middle of the coils, enabling more highly localized stimulation than is possible with the circular coil. By using an array of four or more coil elements over the head, high flexibility in the design of the spatiotemporal pattern of magnetic field pulses becomes possible [Grandori and Ravazzani, 1991; Ruohonen and Ilmoniemi, 1998; Fadini et al., 2009; Lu et al., 2009]. The use of varying three-dimensional configurations of multiple coil elements (such as a slinky coil or a differential coil) can produce more focused stimulation than is possible with planar coils [Lin et al., 2000; Hsu and Durand, 2001], and H-coils can be used to stimulate deeper parts of the brain [Zangen et al., 2005; Roth et al., 2007; Roth et al., 2014]. 
Our previously proposed eccentric figure-eight coil that can provide threshold stimulation to the brain at lower stimulator driving currents [Kato et al., 2012] represents a possible basis for a compact TMS system architecture. In our work described in this paper, we compared the characteristics of an eccentric coil system with those of a conventional coil through numerical simulation, and we fabricated a prototype eccentric coil and then used it to provide magnetic stimulation to healthy subjects. Preliminary results of this study have been reported in conference proceedings [Kato et al., 2011; Sekino et al., 2012].

\section{MATERIALS AND METHODS}

\section{Principles of Eccentric Figure-Eight Coils}

Figure 1 provides a schematic illustration of the winding geometries of both a conventional concentric figure-eight coil and an eccentric figure-eight coil. Whereas the center of the outer circumference coincides with the center of the inner circumference in the concentric coil, in the eccentric coil, the inner circumferences are both shifted toward the tangent point of the two spirals.

In both cases, driving currents flow through the two spirals in opposite directions. When the brain is stimulated by a figure-eight coil, the eddy currents in the brain converge below the middle of the two spirals. As the eccentric coil has denser conductors in its middle, however, a higher eddy current density occurs below these conductors and, as a result, the eccentric coil requires a smaller driving current than the conventional coil to obtain the same eddy current density within the target area. Thus, the eccentric coil can use a driving circuit with a lower output power and, correspondingly, a smaller size; as 
the driving circuit accounts for most of the total weight of a magnetic stimulator system, this provides a considerable advantage in usability.

The discussion above assumes that the inner coil diameter is substantially smaller than the outer diameter. Our preliminary investigations showed that reducing the inner diameter will substantially decrease the coil inductance and thus the voltage required to charge a capacitor in the driving circuit.

\section{Numerical Simulation of Coil Characteristics}

Using numerical simulation, the electromagnetic characteristics of the eccentric coil system were evaluated. Modeling and analyses were carried out using dedicated software (PHOTO-Series: PHOTON Co., Ltd., Kyoto, Japan) for the following analyses of inductances and eddy current distributions in a spherical model. Figure 2(a) shows a half-model of the reference concentric coil used to calculate its inductance (the symmetry in the magnetic field distribution allowed us to extrapolate this half to the entire coil and thus reduce the computation time). To simplify calculations, each spiral winding was modeled as a set of ring conductors $2 \mathrm{~mm}$ wide and $6 \mathrm{~mm}$ high. The outer diameter, inner diameter, and number of turns were $120 \mathrm{~mm}, 20 \mathrm{~mm}$, and 10 , respectively. The two element coils partly overlapped each other with a $0.5 \mathrm{~mm}$ gap; this intensified the convergence of eddy currents below the center of the coil structure and thus increased the maximum eddy current density.

The numerical models of the coils were constructed from tetrahedral finite elements, each of which had an approximate size of $1 \mathrm{~mm}$. Including a $1000 \mathrm{~mm} \times 1000 \mathrm{~mm} \times$ 
$500 \mathrm{~mm}$ analytical region representing the surrounding air, the total number of elements was $5.5 \times 10^{5}$. Magnetic field distributions were then calculated using the finite element method for an electric current of $I=1$ A applied to each ring conductor. At low frequencies, the integration of magnetic field $B$ over the entire volume gives the magnetic field energy, according to the formula $E=\left(1 / 2 \mu_{0}\right) \int B^{2} d V$. Considering the energy conservation, the equal amount of energy is required to apply the current $I$ to the coil with inductance $L$, as expressed by $E=(1 / 2) L I^{2}$. These relations lead to the following equation for estimating the inductance from the calculated magnetic fields,

$$
L=\frac{1}{\mu_{0} I^{2}} \int B^{2} d V
$$

When the thickness of conductor is much larger than the penetration depth, some analytical formulas of inductance are available [Wheeler, 1942]. The pulse waveform of our magnetic stimulator (biphasic pulse with a width of approximately $250 \mu \mathrm{s}$ ) gives the equivalent frequency of $4 \mathrm{kHz}$. Given the conductivity of copper being $6 \times 10^{7} \mathrm{~S} / \mathrm{m}$, the estimated penetration depth of $1 \mathrm{~mm}$ is comparable to the conductor half-thickness of 1 $\mathrm{mm}$. Thus, we adopted the fully-numerical method instead of the analytical formulas.

Eddy current distributions within the human brain were obtained by using the numerical models shown in Figures 2(b) and (c). Again, to take advantage of the symmetry in eddy current distribution, the brain could be modeled as a conductive quarter-sphere surrounded by an insulating layer to mimic the effects of the skull (the skull is not shown in Fig. 2). On the basis of the specifications of the previous work [Lopes da Silva 
et al., 1991; Malmivuo et al., 1997; Chauveau et al., 2004; Gutierrez et al., 2004], we found that the spherical diameter was $144 \mathrm{~mm}$ and the gap between the quarter-sphere and the bottom surface of the coil was $13 \mathrm{~mm}$. The brain model had a uniform electric conductivity of $0.11 \mathrm{~S} / \mathrm{m}$, corresponding to the conductivity of gray matter at $4 \mathrm{kHz}$ [Gabriel et al., 1996]. Altogether, the brain model and insulating consisted of $3.4 \times 10^{5}$ elements. To model the coil operation, $4 \mathrm{kA}$ alternating currents at $4 \mathrm{kHz}$ were applied to each ring conductor in the coil. As the vector potential of the resulting magnetic field produced from the coil was obtained by numerically integrating the Biot-Savart law, a full model of the coil was needed for this calculation. From this, the resulting eddy current distribution in the brain model was obtained using the finite element method [Sekino et al., 2006].

Recent papers have reported eddy current distributions in brain models having realistic anatomy [Sekino et al., 2006; Lu et al., 2008]. Eddy current distributions for the eccentric coils were obtained also for such a head model [Nagaoka et al., 2004]. The model consisted of 2-mm cubic cells segmented into approximately 60 tissues (for the whole body). The conductivities of the tissues were calculated for a frequency of $4 \mathrm{kHz}$ based on Gabriel's dataset [Gabriel et al., 1996]. The stimulator coils were located on the surface of the head so that the middle of the coil was positioned above the primary motor cortex. Electric currents were applied to the coil with a magnitude of $4 \mathrm{kA}$ and a frequency of $4 \mathrm{kHz}$. The vector potential produced from the coil was calculated based on the Biot-Savart law. The eddy current distributions were calculated using an originally-developed software based on the scalar-potential finite-difference method [Dawson and Stuchly, 1996]. 


\section{Fabrication of Prototype Coils}

On the basis of respective concentric and eccentric geometries, prototype magnetic stimulator coils were designed and fabricated; Figures 3(a) and (b) show the winding patterns of the two coils. In the concentric coil, the center of the outer circumference coincides with the center of the inner circumference, whereas in the eccentric coil, the center of each inner circumference is shifted $25 \mathrm{~mm}$ toward the center of the opposing spiral. This decentering resulted in a minimum gap of $0.5 \mathrm{~mm}$ between adjacent conductors at the middle of the two spirals. The blue- and green colors in the Figures represent conductors lying in different winding planes at a gap of $0.5 \mathrm{~mm}$ between overlapping conductors. For an outer diameter, inner diameter, and number of turns of $120 \mathrm{~mm}, 20 \mathrm{~mm}$, and 10, respectively, the eccentric winding trajectory can be defined using,

$$
\begin{aligned}
& x=\left(10+\frac{50}{2 \pi \times 10} \theta\right) \cos \theta \\
& y=\left(10+\frac{50}{2 \pi \times 10} \theta\right) \sin \theta
\end{aligned}
$$

where $x$ and $y$ are in millimeters. The overall winding length is $3.7 \mathrm{~m}$.

Figure 3(c) shows the internal structure of the eccentric coil. Owing to its unique geometry, it was not possible to simply wind the conductor around a bobbin; instead, using numerically controlled processing machinery, we cut eccentric-spiral slits on the insulator cases (which were made from fiberglass-reinforced plastic to provide 
sufficient mechanical strength), and a single core wire with a $2 \mathrm{~mm} \times 6 \mathrm{~mm}$ rectangular cross-section was woven through the slits. This approach enabled us to create arbitrary winding geometries. The case was divided into four parts for easy assembly and featured a $25 \mathrm{~mm}$ diameter and a $130 \mathrm{~mm}$ long handgrip. On the side attached to the human head, the case thickness was $1 \mathrm{~mm}$. Except in details relating to conductor geometry, the concentric coil fabrication process was the same as that for the eccentric coil.

To avert possible thermal damage during repetitive stimulations, each coil was equipped with a temperature sensor consisting of a platinum resistance thermometer made of non-magnetic materials embedded into the base of the handgrip $3 \mathrm{~mm}$ away from the coil conductor (the generated magnetic field was relatively weak at this location).

\section{Magnetic Stimulation to Healthy Subjects}

Magnetic stimulation was performed on the brains of six healthy volunteers. The study protocol was thoroughly reviewed and approved by the ethical committee of Osaka University Hospital (approval number 11173-3).

Three-dimensional MRI of the subjects' brain was obtained beforehand to determine a proper position of the coil. Using an adapter, we connected the coils to a commercially available driving circuit (MagPro R60: MagVenture, Farum, Denmark) that produced biphasic pulse stimulations. The currents flowing in the coil were measured using a current transformer (4418: Pearson Electronics, Palo Alto, CA) connected to an oscilloscope (TDS2024B: Tektronix Inc., Beaverton, OR). The resulting magnetic fields 
were simultaneously measured with a homemade search coil consisting of five $6.3 \mathrm{~mm}$ diameter inner loop turns and four $7.1 \mathrm{~mm}$ diameter outer loop turns (on the basis of these parameters, it was found that the search coil had an effective area of $314 \mathrm{~mm}^{2}$ ). The search coil was located at the center of the inner circumference of one spiral and on the surface of the insulator case. The temperature sensor in the coil was connected to a data logger (ZR-RX45A: Omron Corp., Kyoto, Japan) for continuous monitoring. To receive magnetic stimulation, each subject lay on a bed in the supine position. A proficient neurosurgeon finely positioned the coils above the motor cortex using a navigation system (Brainsight Frameless: Rogure Research, Canada) to stimulate the area corresponding to the abductor pollicis brevis (APB) muscles while watching the muscle reactions to pilot the stimulation. Magnetic stimulations were applied with a single biphasic pulse. Electromyograms of the APB muscles were recorded using an amplifier (Neuropack MEB-2200: Nihon Kohden, Tokyo, Japan). On the basis of this measurement, the neurosurgeon found the stimulus intensity corresponding to the resting motor threshold, which was displayed in units of amperes per microsecond on the front panel of the driving circuit. These stimulations were carried out using both the concentric coil and the eccentric coil on each subject.

\section{RESULTS}

\section{Evaluation of Coil Characteristics from Numerical Results}

Figure 4(a) shows the magnetic field distribution calculated for the concentric coil. Here, the plotting plane is perpendicular to the winding plane and passes through the winding centers of two spirals. The red squares indicate the cross sections of the conductors. This figure shows all the finite model elements, the spacing density of which decreases 
from $1 \mathrm{~mm}$ close to the center to much lower levels in the surrounding air. As the conductors are assumed to carry a unit current of 1 A to estimate the inductance, the generated magnetic field exhibits small flux densities below $1 \mathrm{mT}$. The maximum flux density of $372 \mu \mathrm{T}$ occurs near the innermost conductors and gradually decreases with distance from the conductors. At a conductor current of $4 \mathrm{kA}$, the flux density reached 1.5 $\mathrm{T}-$ a reasonable value in the human brain stimulation.

Figures 4(b) and (c) show the respective eddy current densities of the concentric and eccentric coils. The scale is provided for both eddy current density and electric field intensity. The center of the inner circumference of the eccentric coil shown in Figure 2(c) is shifted by $32 \mathrm{~mm}$ from the center of the outer circumference toward the center of the opposite spiral, completely closing the gap between adjacent conductors at the middle of the two spirals. The current density exhibited a maximum value below the middle of the two coils. In each case, the current density gradually decreased with depth from the surface of the brain model, and the difference in the height of the two winding layers owing to partial overlap resulted in slight asymmetries in the current distributions. Maximum current densities of 18.4 and $22.4 \mathrm{~A} / \mathrm{m}^{2}$ were measured for the concentric and eccentric coils, respectively; the higher current density obtained using the eccentric coil shows the advantage of this coil type.

From these results, it can be calculated that the eccentric coil requires a driving current intensity of approximately $18 \%$ less than that required by the concentric coil in order to obtain a comparable eddy current density in the brain. This economy contributes to reducing Joule loss and thus allows downsizing of the stimulator system; the effect is further enhanced because Joule loss in the coil is proportional to the square of the 
driving current and, therefore, its contribution to reducing heat should be greater than 18\%. In some conditions, such as neuropathic pain, TMS becomes effective when stimulation pulses are delivered at high frequencies above $5 \mathrm{~Hz}$ [Saitoh et al., 2007]. As (all else being equal) repetitive stimulation increases heat generation within the coil, a reduction in the baseline driving-current-related Joule loss enables the use of more compact cooling systems for pulsed TMS, even allowing in some cases the adoption of an air-cooled system instead of a water-cooled system.

Figures 5(a) and (b) show a comparison of eddy current localizations in the concentric and eccentric figure-eight coils. In Figure 5(a), the current densities are sampled along a circular arc following the surface of the brain model. In both cases, the highest eddy current densities appear below the coil centers. In the eddy current maps in Figure 4, the eccentric coil exhibited higher eddy current density at the vertex. In Figure 5, for emphasizing the difference in attenuations, the eddy currents for the two coils are independently scaled so that the values at the vertex become 1.0. It can be seen from this figure that there is a slight difference in distributions between the two coils: in the eccentric coil, the denser conductors in the middle lead to a higher localization of current distribution. In both cases, the direction of eddy currents reverses at approximately $30^{\circ}$ (where the current density becomes zero), and asymmetries in the current distributions are caused by the overlapping structure of each coil.

From Figure 5(b), which shows the dependence of current density on depth from the vertex, it can be seen that the eccentric coil exhibited slightly higher localization, with a current density at $15 \mathrm{~mm}$ from the vertex of approximately half the maximum value on the surface. 
Figure 6 shows the relation of the calculated inductance and the peak eddy current density with the eccentricity factor (the coil models shown in Figs. 2(b) and (c) have eccentricities of 0.0 and 1.0, respectively). It can be seen that both the inductance and peak eddy current density monotonically increase with eccentricity. Assuming a constant pulse width, the observed increase in inductance would necessarily imply a decrease in driving circuit capacitance, and such a capacitance change would increase the charging voltage needed to obtain a comparable stimulus intensity. However, even a voltage increase of $14 \%$ would be within acceptable levels for home patient use.

To calculate the inductance, we considered only the spiral conductors in the coil. The additional inductances in the cables and the driving circuit result in a larger effective inductance for both of the coils.

The results for the realistic head model are presented in Figure 7. The computational cells exhibiting current densities above $25 \mathrm{~A} / \mathrm{m}^{2}$ are indicated red. Figures 7(a) and (b) show the current distributions on the surface of the brain for concentric and eccentric coils, respectively. Comparing these two results, the eccentric coil induced stronger eddy currents in the brain, which was consistent with the results of spherical model. The eddy currents distributed not only within the precentral gyrus but also over adjacent gyri. As shown in Figures 7(c) and (d), the difference in penetration and focality of eddy current was small. While the realistic model can replicate the detailed structure of cerebral gyri and sulci, the effect of coil design on eddy current was partly perturbed. The simplified analyses using the spherical model more clearly demonstrate the influence of coil design parameters. 


\section{Performance of Prototype Coils}

Prior to assembly, coil conductors were wound into roughly spiral-shaped forms and were then inserted into spiral slits carved onto the insulator cases, as shown in Figure 8(a). After assembling the conductor, insulator case, and temperature sensor, the internal void spaces were filled with epoxy to provide mechanical support and electrical insulation. A flexible $1.5 \mathrm{~m}$ long cable was connected to the terminals of the coil, and then, a handgrip was attached to the coil body. Figure 8(b) shows the finished eccentric coil. The coil body weighed approximately $1.8 \mathrm{~kg}$ and thus could be easily manipulated with one hand.

For evaluating the withstanding voltage, a static voltage of $4 \mathrm{kV}$ was applied for $1 \mathrm{~min}$ between the coil conductors and the surface of the insulator case using a breakdown test device (TOS8750: KIKUSUI Electronics Corp., Yokohama, Japan). This value of voltage is more than double the voltage applied to the coils during magnetic stimulation to human subjects. No dielectric breakdown was detected in the insulator case.

The coil inductances_-including those of the cables_-were measured using an inductance/capacitance/resistance (LCR) meter (3522-50: HIOKI E.E. Corp., Nagano, Japan) with an operating frequency of $4 \mathrm{kHz}$ and were found to be 11.9 and $12.9 \mu \mathrm{H}$ in the concentric and eccentric coils, respectively. After this, pulsed currents of up to $5 \mathrm{kA}$ were applied to the coils using a custom-built pulsed power supply with a pulse width of approximately $240 \mu$ s to ensure that the transient electromagnetic forces caused no damage to the insulator case. 


\section{Resting Motor Thresholds of Healthy Subjects}

The pulsed currents flowing in the developed coils and the resulting magnetic fields were measured using the current transformer and the search coil, respectively. The current slew rate was $80 \mathrm{~A} / \mu$ s for both coils, corresponding to approximately $56 \%$ of the maximum output of the driving circuit. Figure 9(a) shows the recorded currents for the two coils. The pulse width of the eccentric coil $(302 \mu \mathrm{s})$ was longer than that of the concentric coil ( $292 \mu \mathrm{s})$ because of the larger inductance of the eccentric coil. Given the aforementioned equal slew rate for both coils and these pulse widths, the peak current of the eccentric coil (3.48 kA) was higher than that of the concentric coil (3.32 kA). The waveforms recorded using the search coil are shown in Figure 9(b). Numerical integration of the waveforms resulted in peak magnetic flux densities of 721 and 802 $\mathrm{mT}$ for the concentric and eccentric coils, respectively. The ratios of magnetic flux density to coil current, $217 \mathrm{mT} / \mathrm{kA}$ for the concentric coil and $230 \mathrm{mT} / \mathrm{kA}$ for the eccentric coil, provide a supportive evidence for the higher efficacy of the eccentric coil.

Given the coil resistance of approximately $22 \mathrm{~m} \Omega$ at $4 \mathrm{kHz}$ for both coils, the Joule heating was estimated to be $3.5 \mathrm{~J}$ per pulse. For evaluating the heat generation, the coil temperature was monitored using the embedded sensor while applying 500 pulses in 10 minutes. The coil temperature increased from 27.0 to $34.7^{\circ} \mathrm{C}$.

Figure 10(a) shows an electromyogram recording from the APB muscles of one subject. On the plot, the TMS pulse is applied at $32 \mathrm{~ms}$. The first peak of the myoelectric 
potential is observed to have a latency of approximately $15 \mathrm{~ms}$ after the TMS pulse, and the peak potential is approximately $20 \mu \mathrm{V}$, corresponding to barely visible muscle contraction.

Figure 10(b) shows the averages and standard deviations of stimulus intensities corresponding to resting motor thresholds for concentric and eccentric spiral coils. For all six subjects, determination of the motor threshold was successfully carried out. The motor thresholds for the concentric and eccentric coils were 86 and $78 \mathrm{~A} / \mu \mathrm{s}$, respectively. The lower motor threshold demonstrated by the eccentric coil is statistically significant for a $p$ value smaller than 0.01 in a paired Student's $t$-test. Although owing to a relatively large variation in the motor thresholds between subjects the error bars in Figure 10(b) overlap, the eccentric coil exhibited lower motor thresholds for all six subjects, resulting in the statistically significant difference. There results indicate that the eccentric coil consistently requires a lower driving current to reach the motor threshold than does the concentric coil. The peak currents corresponding to the motor thresholds for the concentric and eccentric coils were 3.6 and $3.4 \mathrm{kA}$, respectively. The pulse powers estimated from the peak currents and inductances were $77 \mathrm{~J}$ for the concentric coil and $75 \mathrm{~J}$ for the eccentric coil.

\section{DISCUSSION}

Numerical simulations of eddy currents in TMS have been reported in many papers. A pioneering study was performed by Tofts using a single circular coil and a conductor with planar surface [Tofts, 1990]. His parameters, coil current of $10^{8} \mathrm{~A} / \mathrm{s}$ and tissue conductivity of $0.2 \mathrm{~S} / \mathrm{m}$, resulted in the peak eddy current of $6.8 \mathrm{~A} / \mathrm{m}^{2}$. Our simulations 
were performed with the coil current of $2.0 \times 10^{9} \mathrm{~A} \cdot$ Turn/s and tissue conductivity of $0.11 \mathrm{~S} / \mathrm{m}$, and resulted in the peak eddy current of $22 \mathrm{~A} / \mathrm{m}^{2}$. Considering that the eddy current is proportional to the coil current and inversely proportional to the conductivity, introducing Tofts's parameters into our simulations would give an eddy current of 2 $\mathrm{A} / \mathrm{m}^{2}$. This value is smaller than his result but within the same order of magnitude. The difference is attributable to the coil design and conductor geometry. While Tofts's model introduces the coil current concentrated in single wire and the conductor having planar surface, our model introduces the coil current distributed in 20 wires and the conductor having spherical surface.

In our studies, we formed an eccentric winding design on a plane that allows for easy fabrication; however, an eccentric coil can also be formed on a curved surface, such as a cylinder or a sphere, to better fit the shape of the human head. The resulting smaller gap between the coil conductors and the brain would further decrease the coil current required to reach the motor threshold, and a previous study suggests that curved winding leads to lower localization in eddy currents [Lontis et al., 2006].

Stimulations to the primary motor cortex are effective in alleviating the neuropathic pain. Because in general a localized stimulation is desirable to avoid stimulating other brain areas and thus reduce the possibility of adverse side effects, the TMS treatment has been carried out using figure-eight coils with highly localizing eddy currents. Patients in home treatment will not necessarily be able to accurately position the coil on their motor cortex. To address this issue, our group is developing a navigation system to aid patients in self-positioning the coil [Okada et al., 2012; Yasumuro et al., 2012]. A preliminary study has shown that this system can limit the error from the optimal 
position to less than $5 \mathrm{~mm}$ and $5^{\circ}$, suggesting that a suitable current localization would be greater than $5 \mathrm{~mm}$

According to previous studies, the use of an iron core in a stimulator coil is effective in reducing the required driving current [Epstein and Davey, 2002; Han et al., 2003]. However, an iron core will increase the weight of the stimulator coil as well as the hysteresis loss from eddy currents within the core; thus, a heavy coil may not be suitable for finely adjusting the coil position. As our prototype coil is not equipped with an iron core, the weight is kept as small as possible.

In addition to neuropathic pain, we are currently examining the use of our compact magnetic stimulator for the treatment of intractable neurological diseases. Recent studies have shown that TMS is effective for treating Parkinson's disease and depression [Pascual-Leone et al., 1994; Lomarev et al., 2006; Padberg and George, 2009; Maruo et al., 2013], and the prospect of conducting TMS treatment at home is a promising one for combating these diseases. While medical evidences about the efficacy of TMS treatment for these neurological diseases have been established, the underlying mechanism of TMS still remains to be understood. For TMS to the motor cortex, neurophysiological studies have suggested that TMS induces excitations in axons of cortical interneurons which run parallel to the surface of the cortex [Mano et al., 1993]. The excitations are then transmitted to pyramidal cells to activate the motor system. Physical models of neuronal excitation in the central nervous system have recently been proposed to support these understandings of mechanism [Pashut et al., 2011]. Macroscopic imaging of evoked activities using magnetic resonance imaging or positron emission tomography should also be important. The insights into the 
mechanism of magnetic stimulation may be relevant for the design and construction of stimulator coils, and may aid the interpretation of results of TMS of the central nervous system.

The pulse power applied to the coil is limited based on physiological considerations rather than electrical/mechanical factors. Most of the repetitive TMS is carried out with intensities below the motor threshold ( $78 \mathrm{~A} / \mu$ s for the eccentric coil) because muscle contraction may cause discomfort or risk of injury of patients. Based on the established guidelines for the use of TMS in clinical practice [Rossi et al., 2009], it should be possible to safely conduct TMS in patients' homes. By adjusting the intensity of stimulation to suitably limit the risk of inducing seizures while providing substantial therapeutic effects, TMS can be made as safe as electrical stimulation, which is already being carried out at home.

\section{CONCLUSIONS}

Numerical simulations using both simplified and realistic brain models showed that the eccentric coil required a driving current intensity less than that required by the concentric coil to cause comparable eddy current densities within the brain. In magnetic stimulation to healthy subjects, the current slew rate corresponding to motor threshold values for the concentric and eccentric coils were $86 \mathrm{~A} / \mu \mathrm{s}$ and $78 \mathrm{~A} / \mu \mathrm{s}$, respectively. These results showed the advantage of the eccentric coil for the use in a compact stimulator. Future development and achieving the practical use of compact TMS systems will enable the treatment of some intractable neurological diseases at home. 


\section{REFERENCES}

Barker AT, Jalinous R, Freeston IL. 1985. Non-invasive magnetic stimulation of human motor cortex. Lancet 1:1106-1107.

Bischoff C, Machetanz J, Meyer BU, Conrad B. 1994. Repetitive magnetic nerve stimulation: technical considerations and clinical use in the assessment of neuromuscular transmission. Electroencephalogr Clin Neurophysiol 93:15-20.

Chauveau N, Franceries X, Doyon B, Rigaud B, Morucci JP, Celsis P. 2004. Effects of skull thickness, anisotropy, and inhomogeneity on forward EEG/ERP computations using a spherical three-dimensional resistor mesh model. Hum Brain Mapp 21:86-97.

Dawson TW, Stuchly MA. 1996. Analytic validation of a three-dimensional scalar-potential finite-difference code for low-frequency magnetic induction. Appl Comput Electromagn Soc J 11:72-81.

De Sauvage RC, Beuter A, Lagroye I, Veyret B. 2010. Design and construction of a portable transcranial magnetic stimulation (TMS) apparatus for migraine treatment. J Med Devices 4:015002-1-6.

Epstein CM, Davey KR. 2002. Iron-core coils for transcranial magnetic stimulation. J Clin Neurophysiol 19:376-381.

Epstein CM. 2008. A six-pound battery-powered portable transcranial magnetic stimulator. Brain Stimul 1:128-130.

Fadini T, Matthäus L, Rothkegel H, Sommer M, Tergau F, Schweikard A, Paulus W, Nitsche MA. 2009. H-coil: Induced electric field properties and input/output curves on healthy volunteers, comparison with a standard figure-of-eight coil. Clin Neurophysiol 120:1174-1182. 
Gabriel S, Lau RW, Gabriel C. 1996. The dielectric properties of biological tissues: II. Measurements in the frequency range $10 \mathrm{~Hz}$ to $20 \mathrm{GHz}$. Phys Med Biol 41:2251-2269.

Grandori F, Ravazzani P. 1991. Magnetic stimulation of the motor cortex: Theoretical considerations. IEEE Trans Biomed Eng 38:180-191.

Gutierrez D, Nehorai A, Muravchik CH. 2004. Estimating brain conductivities and dipole source signals with EEG arrays. IEEE Trans Biomed Eng 51:2113-2122.

Han BH, Lee SY, Kim JH, Yi JH. 2003. Some technical aspects of magnetic stimulation coil design with the ferromagnetic effect. Med Biol Eng Comput 41:516-518.

Hirayama A, Saitoh Y, Kishima H, Shimokawa T, Oshino S, Hirata M, Kato A, Yoshimine T. 2006. Reduction of intractable deafferentation pain by navigation-guided repetitive transcranial magnetic stimulation of the primary motor cortex. Pain 122:22-27.

Hsu KH, Durand DM. 2001. A 3-D differential coil design for localized magnetic stimulation. IEEE Trans Biomed Eng 48:1162-1168.

Kato T, Sekino M, Matsuzaki T, Nishikawa A, Saitoh Y, Ohsaki H. 2011. Fabrication of a prototype magnetic stimulator equipped with eccentric spiral coils. Proc IEEE Eng Med Biol Soc 1985-1988.

Kato T, Sekino M, Matsuzaki T, Nishikawa A, Saitoh Y, Ohsaki H. 2012. Electromagnetic characteristics of eccentric figure-eight coils for transcranial magnetic stimulation: A numerical study. J Appl Phys 111:07B322.

Lomarev MP, Kanchana S, Bara-Jimenez W, Iyer M, Wassermann EM, Hallett M. 2006. Placebo-controlled study of rTMS for the treatment of Parkinson's disease. Mov Disord 21:325-331.

Lopes da Silva FH, Wieringa HJ, Peters MJ. 1991. Source localization of EEG versus 
MEG: Empirical comparison using visually evoked responses and theoretical considerations. Brain Topogr 4:133-142.

Lin VW, Hsiao IN, Dhaka V. 2000. Magnetic coil design considerations for functional magnetic stimulation. IEEE Trans Biomed Eng 47:600-610.

Lontis ER, Voigt M, Struijk JJ. 2006. Focality assessment in transcranial magnetic stimulation with double and cone coils. J Clin Neurophysiol 23:463-472.

Lu M, Ueno S, Thorlin T, Persson M. 2008. Calculating the activating function in the human brain by transcranial magnetic stimulation. IEEE Trans Magn $44: 1438-1441$.

Lu M, Ueno S, Thorlin T, Persson M. 2009. Calculating the current density and electric field in human head by multichannel transcranial magnetic stimulation. IEEE Trans Magn 45:1662-1665.

Malmivuo J, Suihko V, Eskola H. 1997. Sensitivity distributions of EEG and MEG measurements. IEEE Trans Biomed Eng 44:196-208.

Mano Y, Morita Y, Tamura R, Morimoto S, Takayanagi T, Mayer RF. 1993. The site of action of magnetic stimulation of human motor cortex in a patient with motor neuron disease. J Electromyogr Kinesiol 3:245-250.

Maruo T, Hosomi K, Shimokawa T, Kishima H, Oshino S, Morris S, Kageyama Y, Yokoe M, Yoshimine T, Saitoh Y. 2013. High-frequency repetitive transcranial magnetic stimulation over the primary foot motor area in Parkinson's disease. Brain Stimul 6:884-891.

Nagaoka T, Watanabe S, Sakurai K, Kunieda E, Watanabe S, Taki M, Yamanaka Y. 2004. Development of realistic high-resolution whole-body voxel models of Japanese adult males and females of average height and weight, and application of models to radio-frequency electromagnetic-field dosimetry. Phys Med Biol 49:1-15. 
Okada A, Nishikawa A, Fukushima T, Taniguchi K, Miyazaki F, Sekino M, Yasumuro Y, Matsuzaki T, Hosomi K, Saitoh Y. 2012. Magnetic navigation system for home use of repetitive transcranial magnetic stimulation (rTMS). Proc ICME Int Conf Complex Med Eng 112-118.

Padberg F, George MS. Repetitive transcranial magnetic stimulation of the prefrontal cortex in depression. 2009. Exp Neurol 219:2-13.

Pascual-Leone A, Valls-Sole J, Brasil-Neto JP, Cammarota A, Grafman J, Hallett M. 1994. Akinesia in parkinson's disease: II. Effects of subthreshold repetitive transcranial motor cortex stimulation. Neurology 44:892-898.

Pashut T, Wolfus S, Friedman A, Lavidor M, Bar-Gad I, Yeshurun Y, Korngreen A. 2011. Mechanisms of Magnetic Stimulation of Central Nervous System Neurons. PLoS Comput Biol 7:e1002022.

Rossi S, Hallett M, Rossini PM, Pascual-Leone A. 2009. Safety of TMS Consensus Group, Safety, ethical considerations, and application guidelines for the use of transcranial magnetic stimulation in clinical practice and research. Clin Neurophysiol 120:2008-2039.

Roth Y, Amir A, Levkovitz Y, Zangen A. 2007. Three-dimensional distribution of the electric field induced in the brain by transcranial magnetic stimulation using figure-8 and deep H-coils. J Clin Neurophysiol 24:31-38.

Roth Y, Levkovitz Y, Pell GS, Ankry M, Zangen A. 2014. Safety and characterization of a novel multi-channel TMS stimulator. Brain Stimul 7:194-205.

Ruohonen J, Ilmoniemi RJ. 1998. Focusing and targeting of magnetic brain stimulation using multiple coils. Med Biol Eng Comput 36:297-301.

Saitoh Y, Shibata M, Hirano S, Hirata M, Mashimo T, Yoshimine T. 2000. Motor cortex stimulation for central and peripheral deafferentation pain: Report of eight cases. 
J Neurosurg 92:150-155.

Saitoh Y, Hirayama A, Kishima H, Shimokawa T, Oshino S, Hirata M, Tani N, Kato A, Yoshimine T. 2007. Reduction of intractable deafferentation pain due to spinal cord or peripheral lesion by high-frequency repetitive transcranial magnetic stimulation of the primary motor cortex. J Neurosurg 107:555-559.

Sekino M, Hirata M, Sakihara K, Yorifuji S, Ueno S. 2006. Intensity and localization of eddy currents in transcranial magnetic stimulation to the cerebellum. IEEE Trans Magn 42:3575-3577.

Sekino M, Kato T, Ohsaki H, Saitoh Y, Matsuzaki T, Nishikawa A. 2012. Eccentric figure-eight magnetic stimulator coils. Proc ICME Int Conf Complex Med Eng 728-733.

Tofts PS. 1990. The distribution of induced currents in magnetic stimulation of the nervous system. Phys Med Biol 35:1119-1128.

Tsubokawa T, Katayama Y, Yamamoto T, Hirayama T, Koyama S. 1991. Treatment of thalamic pain by chronic motor cortex stimulation. Pacing Clin Electrophysiol $14: 131-134$.

Ueno S, Tashiro T, Harada K. 1988. Localized stimulation of neural tissues in the brain by means of a paired configuration of time-varying magnetic fields. J Appl Phys 64:5862-5864.

Ueno S, Matsuda T, Fujiki M. 1990. Functional mapping of the human motor cortex obtained by focal and vectorial magnetic stimulation of the brain. IEEE Trans Magn 26:1539-1544.

Weyh T, Wendicke K, Mentschel C, Zantow H, Siebner HR. 2005. Marked differences in the thermal characteristics of figure-of-eight shaped coils used for repetitive transcranial magnetic stimulation. Clin Neurophysiol 116:1477-1486. 
Wheeler HA. 1942. Formulas for the skin effect. Proc IRE 412-424.

Yasumuro Y, Hosomi K, Saitoh Y, Matsuzaki T. 2012. Uncertainty assessment of target localization for rTMS treatment. Proc ICME Int Conf Complex Med Eng 784-787.

Zangen A, Roth Y, Voller B, Hallett M. 2005. Transcranial magnetic stimulation of deep brain regions: Evidence for efficacy of the H-coil. Clin Neurophysiol 116:775-779. 


\section{Figure Captions}

\section{Fig. 1:}

Coil designs and eddy current pathways of (a) a conventional figure-eight coil and (b) an eccentric figure-eight coil.

\section{Fig. 2:}

(a) Numerical model of a concentric coil for estimating the inductance. (b) Models of a concentric coil and the brain for evaluating eddy current distribution in the brain. (c) Models of an eccentric coil and the brain.

\section{Fig. 3:}

(a) Conductors in a prototype concentric coil. (b) Conductors in an eccentric coil. (c) Structure of the conductor and the insulator case.

\section{Fig. 4:}

(a) Magnetic field distribution on the middle section of the concentric coil with unit current flowing in the conductors. (b) Eddy current distribution in the spherical brain model induced with the concentric coil. (c) Eddy current distribution induced with the eccentric coil.

\section{Fig. 5:}

(a) Eddy current distributions in the brain model as a function of angle with vertical. The current density was normalized with the maximum value. (b) Normalized current distributions as a function of depth from the top of the brain model. 


\section{Fig. 6:}

Dependence of the maximum eddy current density in the brain model and the inductance on the eccentricity factor of coil. The concentric coil has an eccentricity factor of zero. The factor takes a value of 1.0 when the ring conductors touch each other in the middle of the coil.

\section{Fig. 7:}

(a) Eddy current distribution on the surface of realistic human brain model induced with the concentric coil. (b) Eddy current distribution induced with the eccentric coil. (c)(d) Eddy current distributions on a coronal section for the two coils.

\section{Fig. 8:}

(a) Formation of an eccentric spiral coil on a plastic insulator case. (b) Finished eccentric coil equipped with a grip and a lead cable. Coil conductors are observed through a 1-mm-thick insulator.

\section{Fig. 9:}

Magnetic fields generated from the fabricated eccentric coil and the MagPro R60 driving circuit. The inset image shows a search coil.

\section{Fig. 10:}

(a) Electromyogram induced with TMS. The potential was recorded from the left abductor pollicis brevis muscles of one subject. (b) Resting motor thresholds for concentric and eccentric coils. 
Fig.1

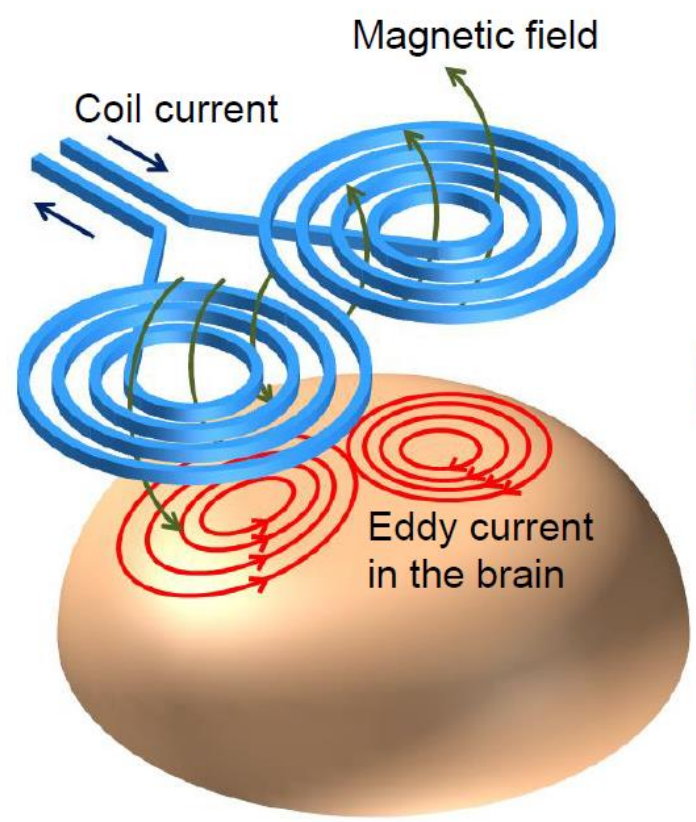

(a)

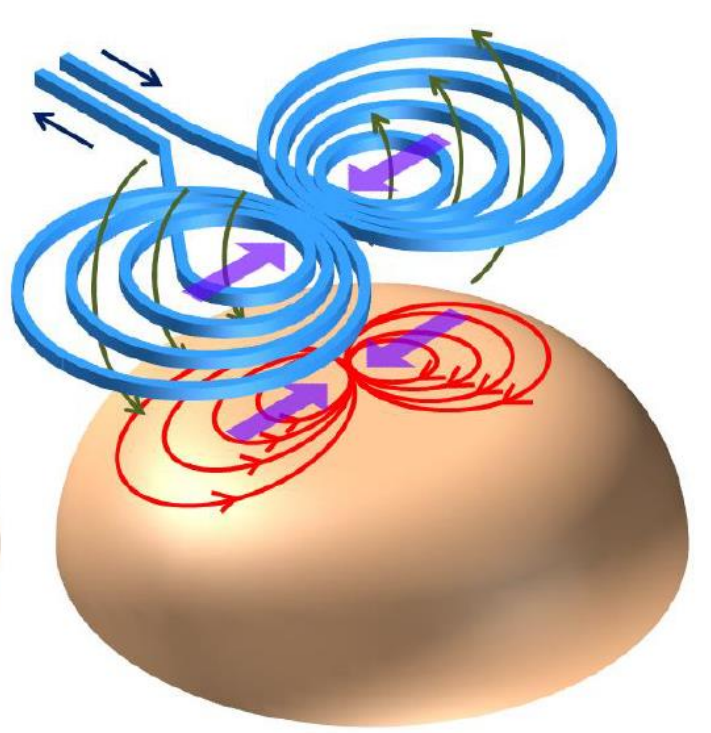

(b)

Fig.2
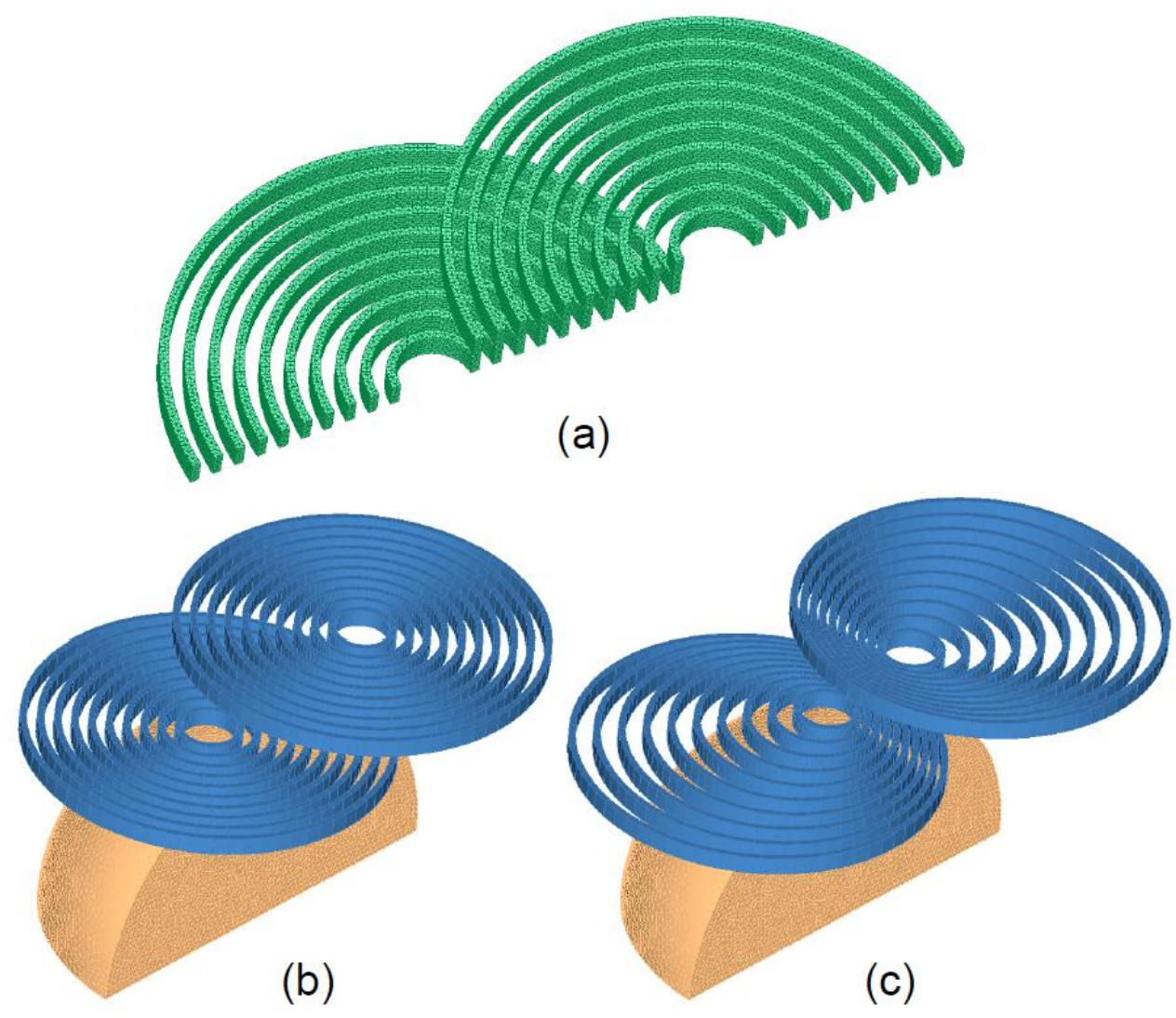
Fig.3

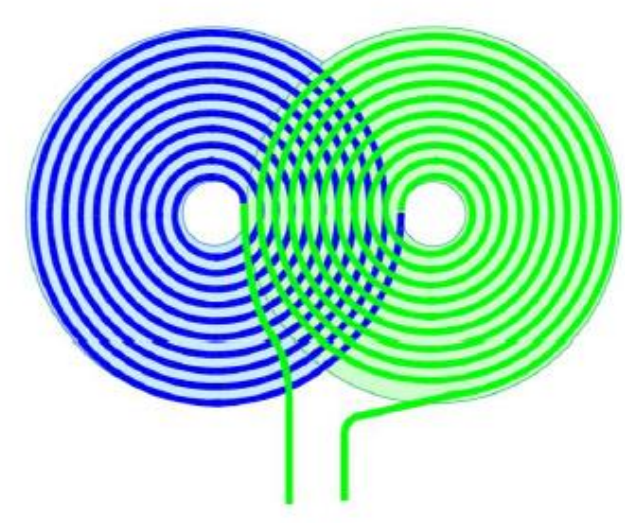

(a)

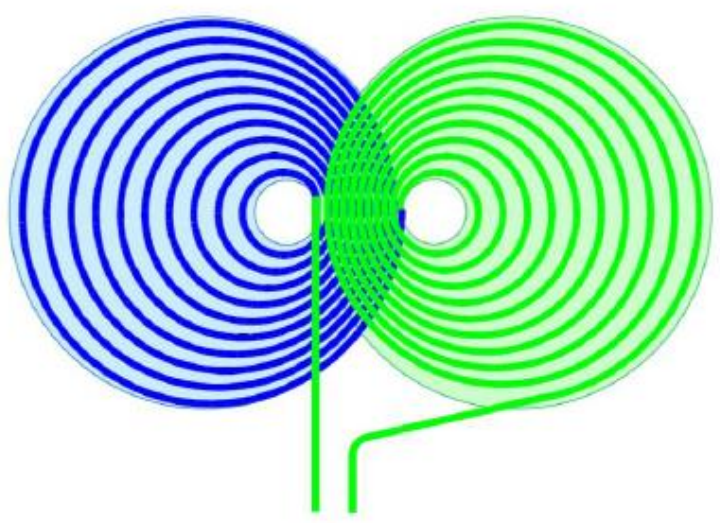

(b)

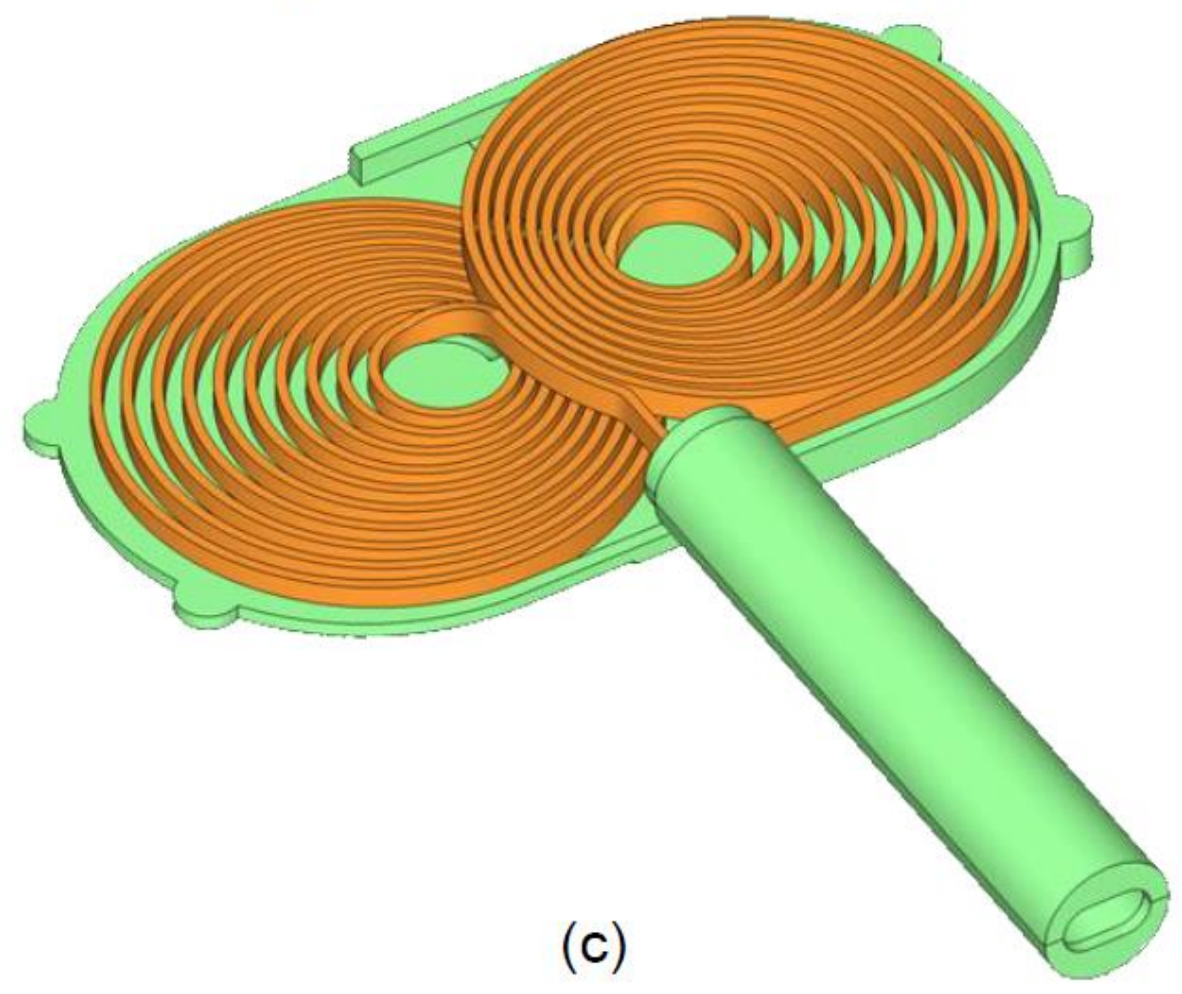


Fig.4

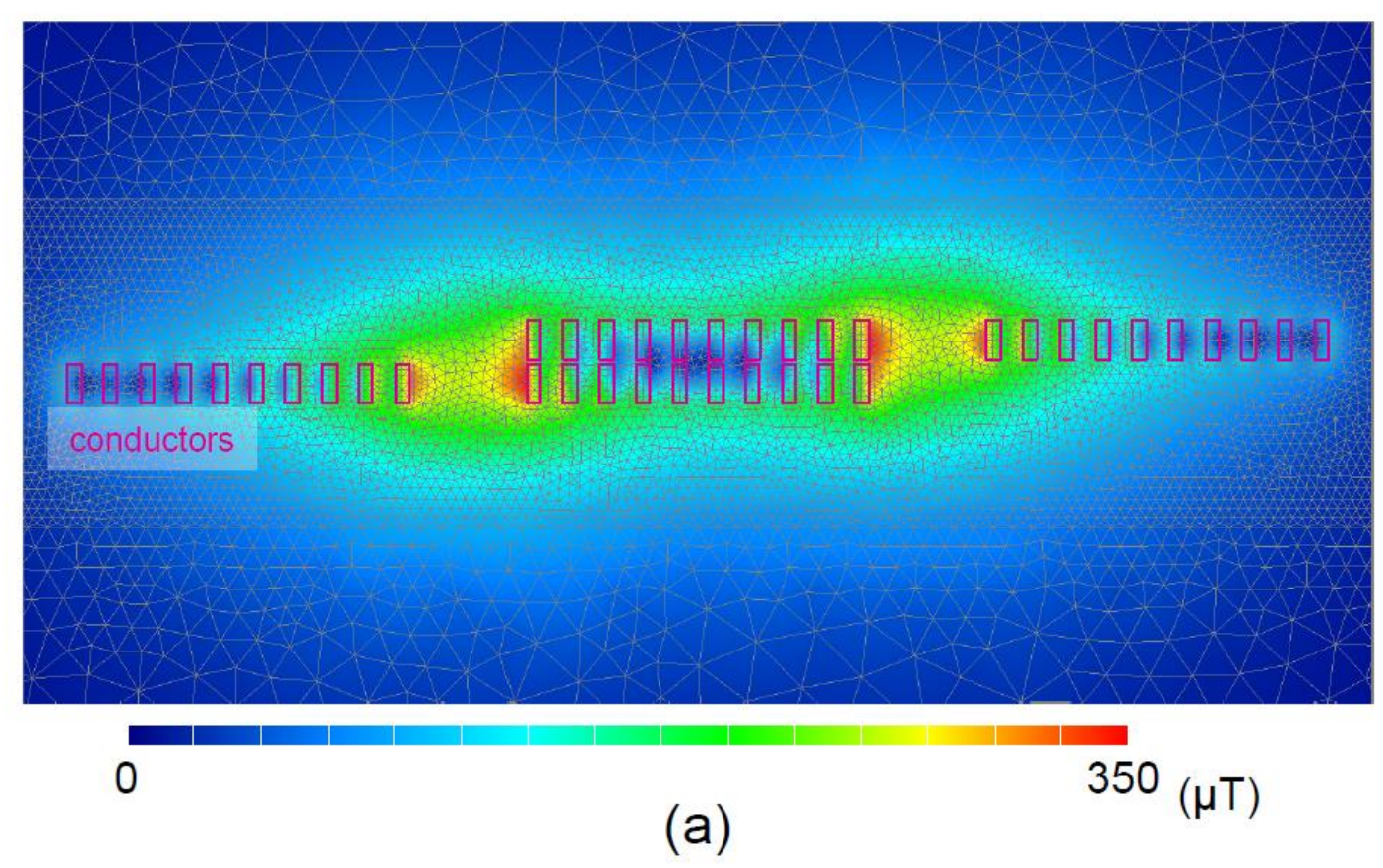

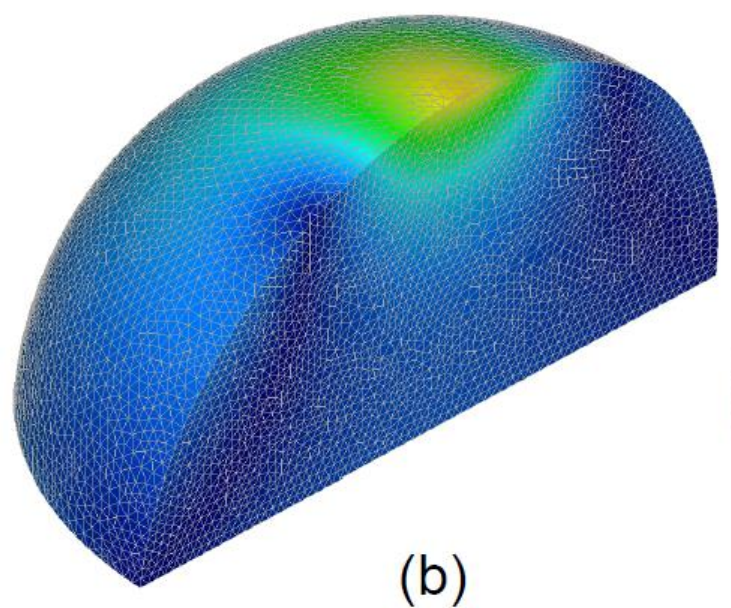

(b)
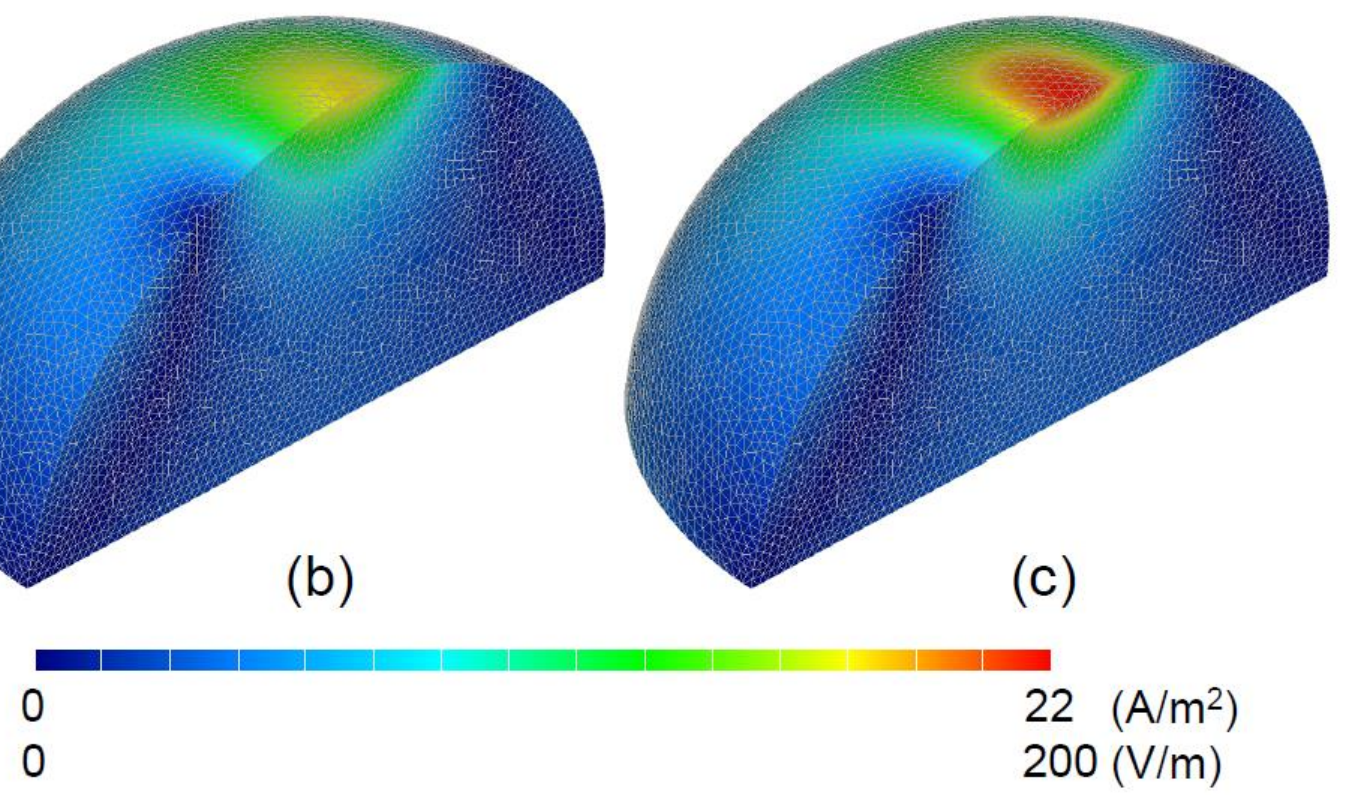


\section{Fig.5}

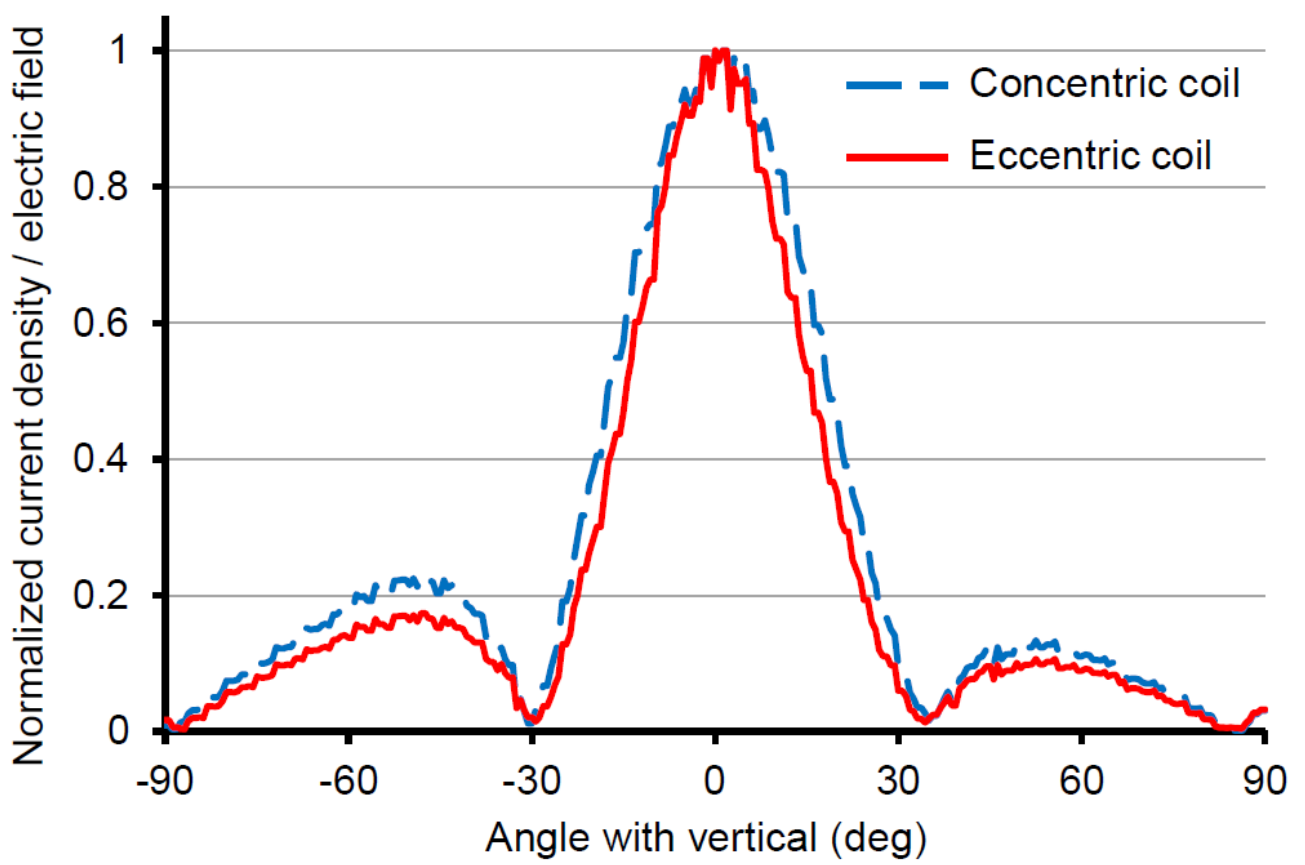

(a)

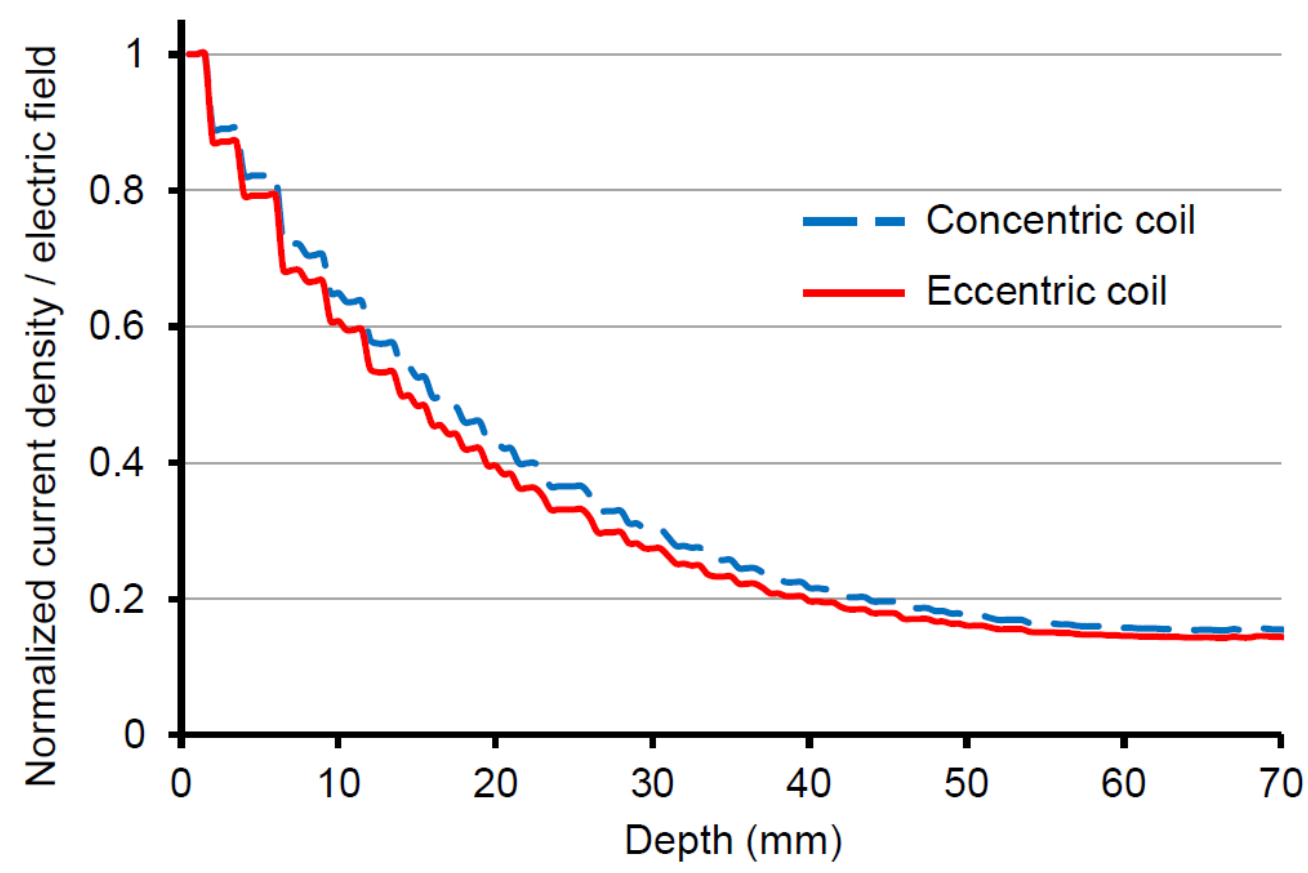

(b) 
Fig.6

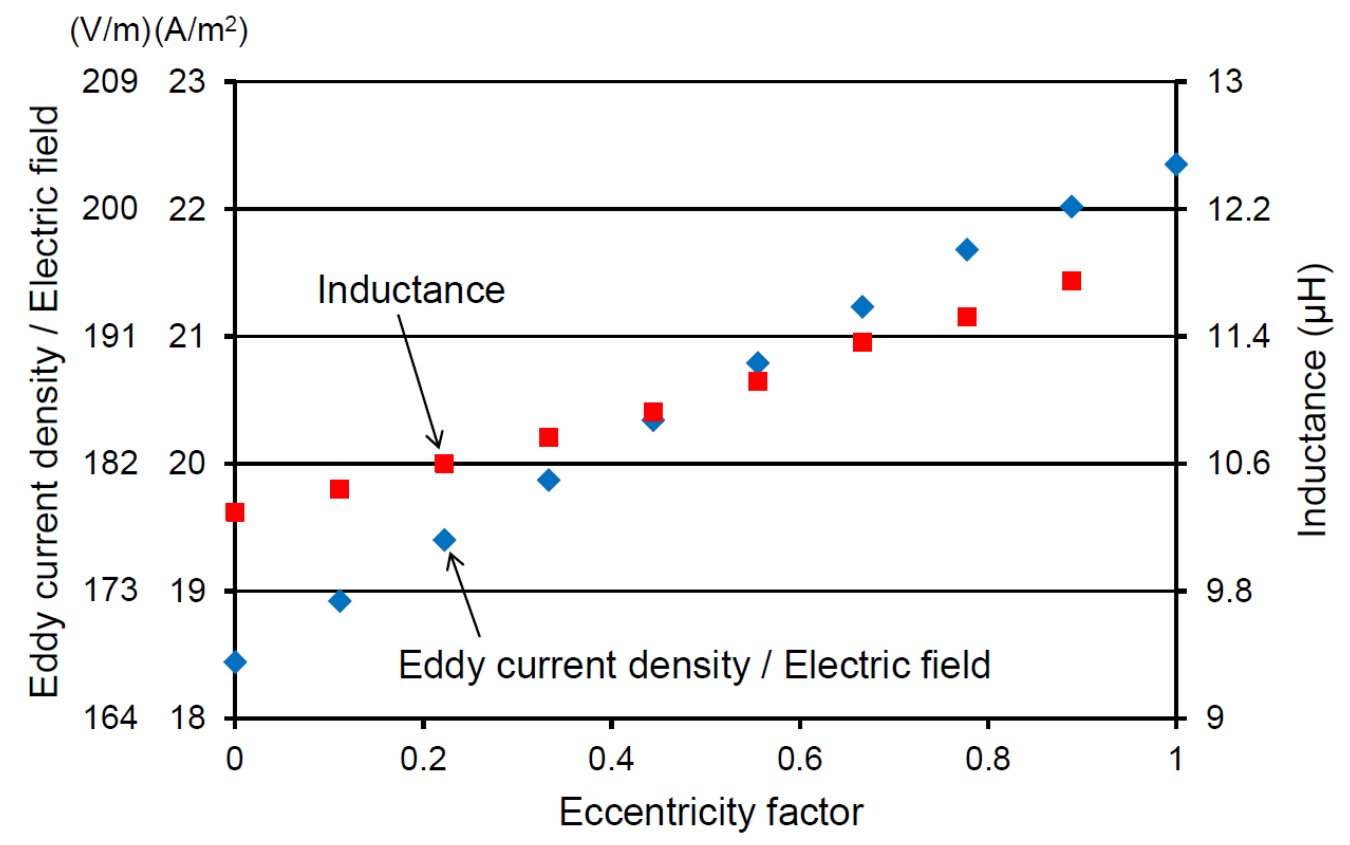


Fig.7

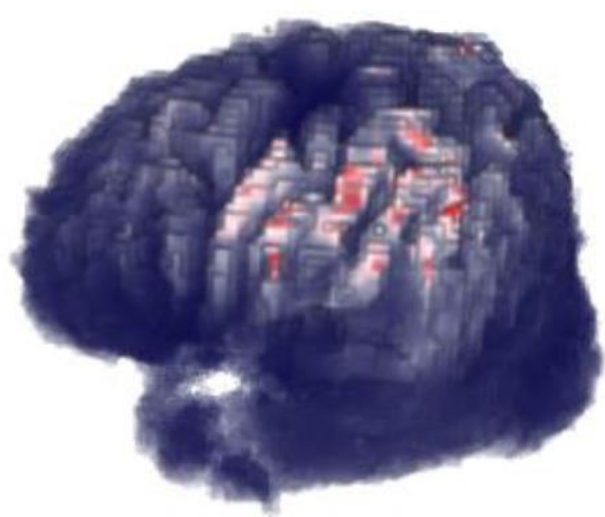

(a)

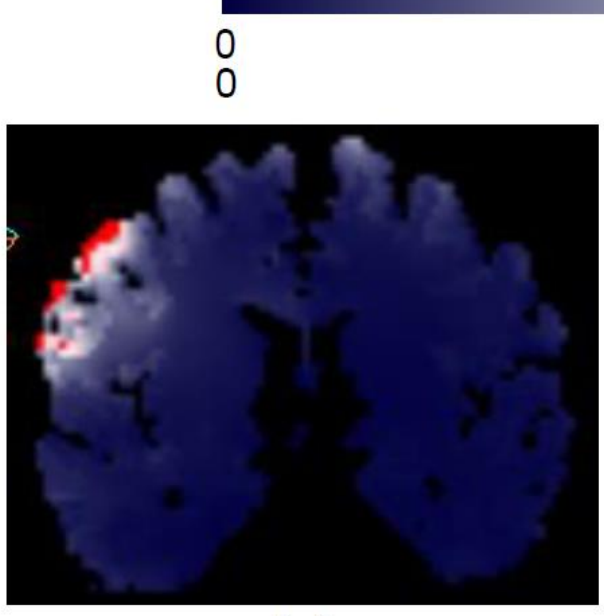

(c)

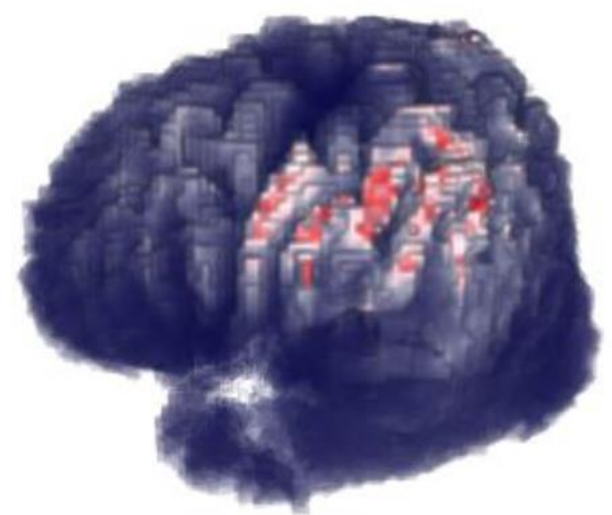

(b)
$25\left(\mathrm{~A} / \mathrm{m}^{2}\right)$

$227(\mathrm{~V} / \mathrm{m})$

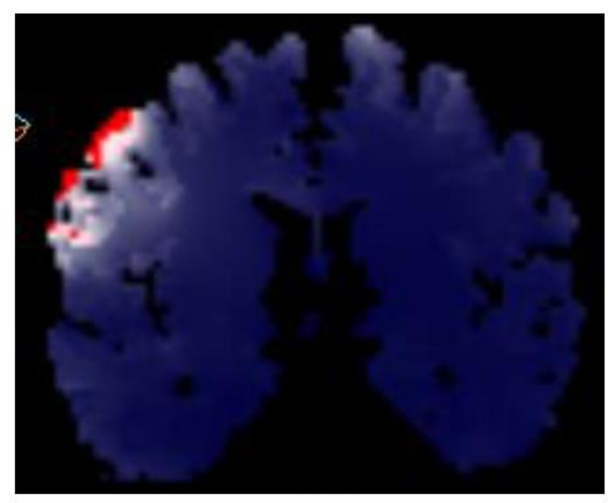

(d) 
Fig.8

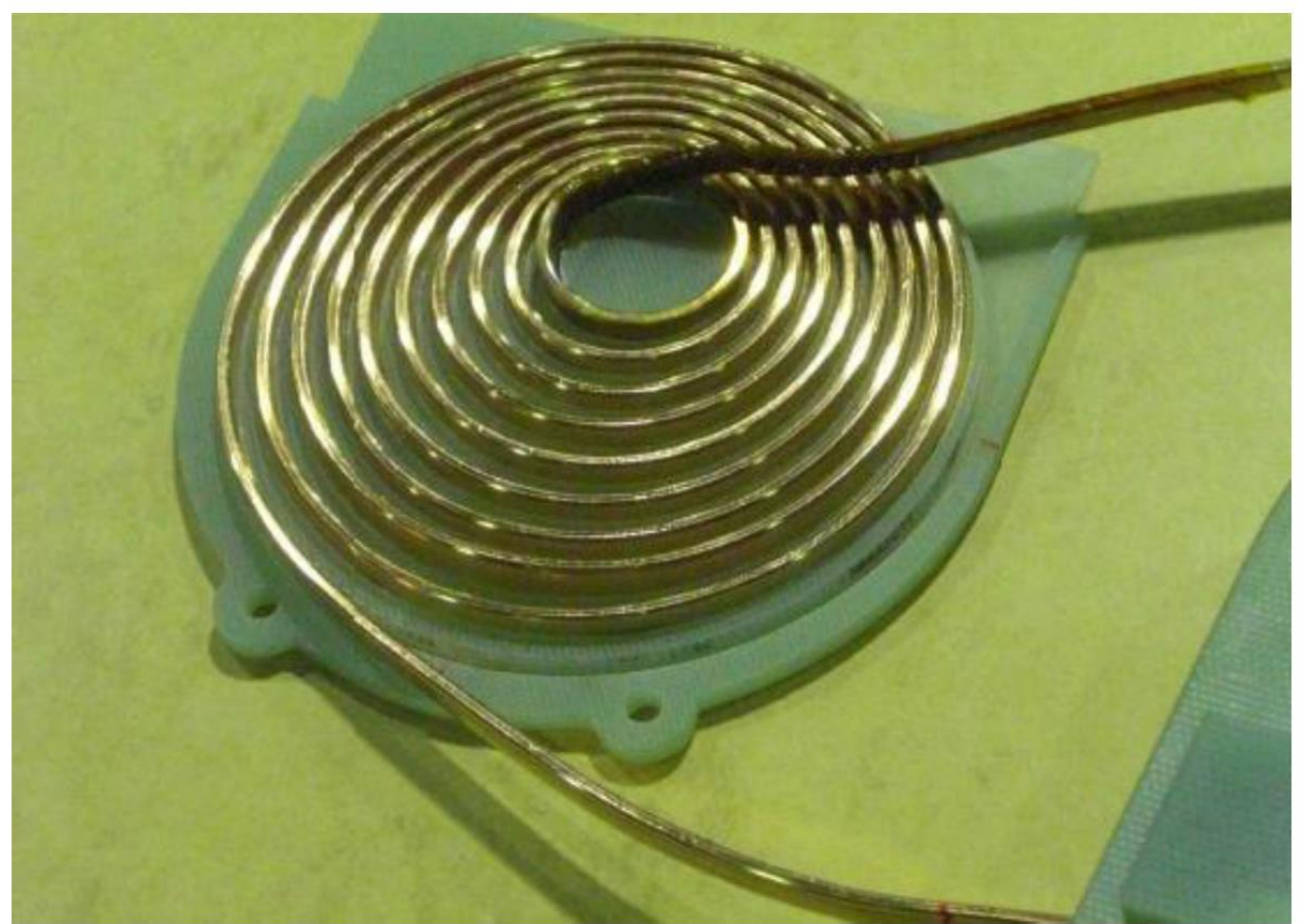

(a)

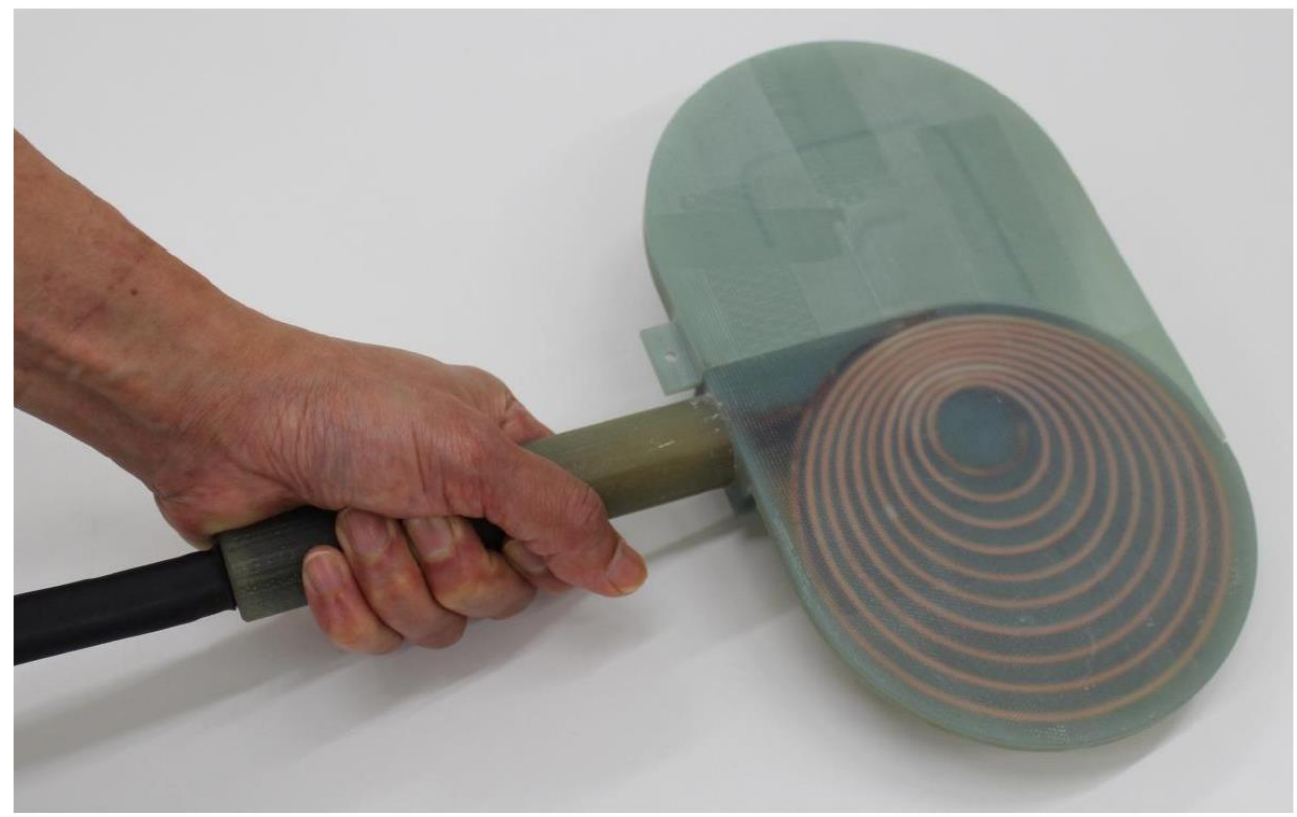

(b) 


\section{Fig.9}
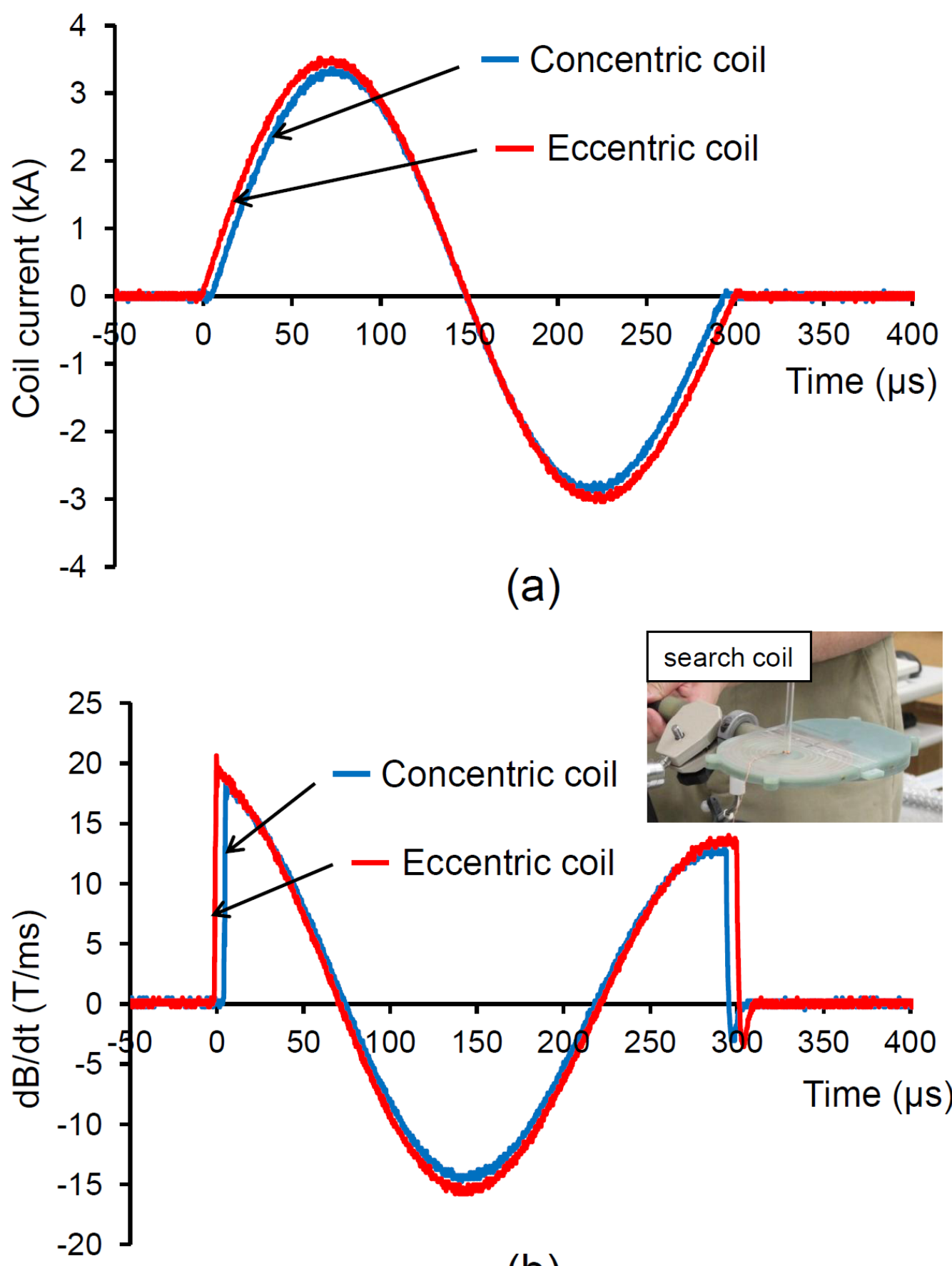

(b) 


\section{Fig.10}
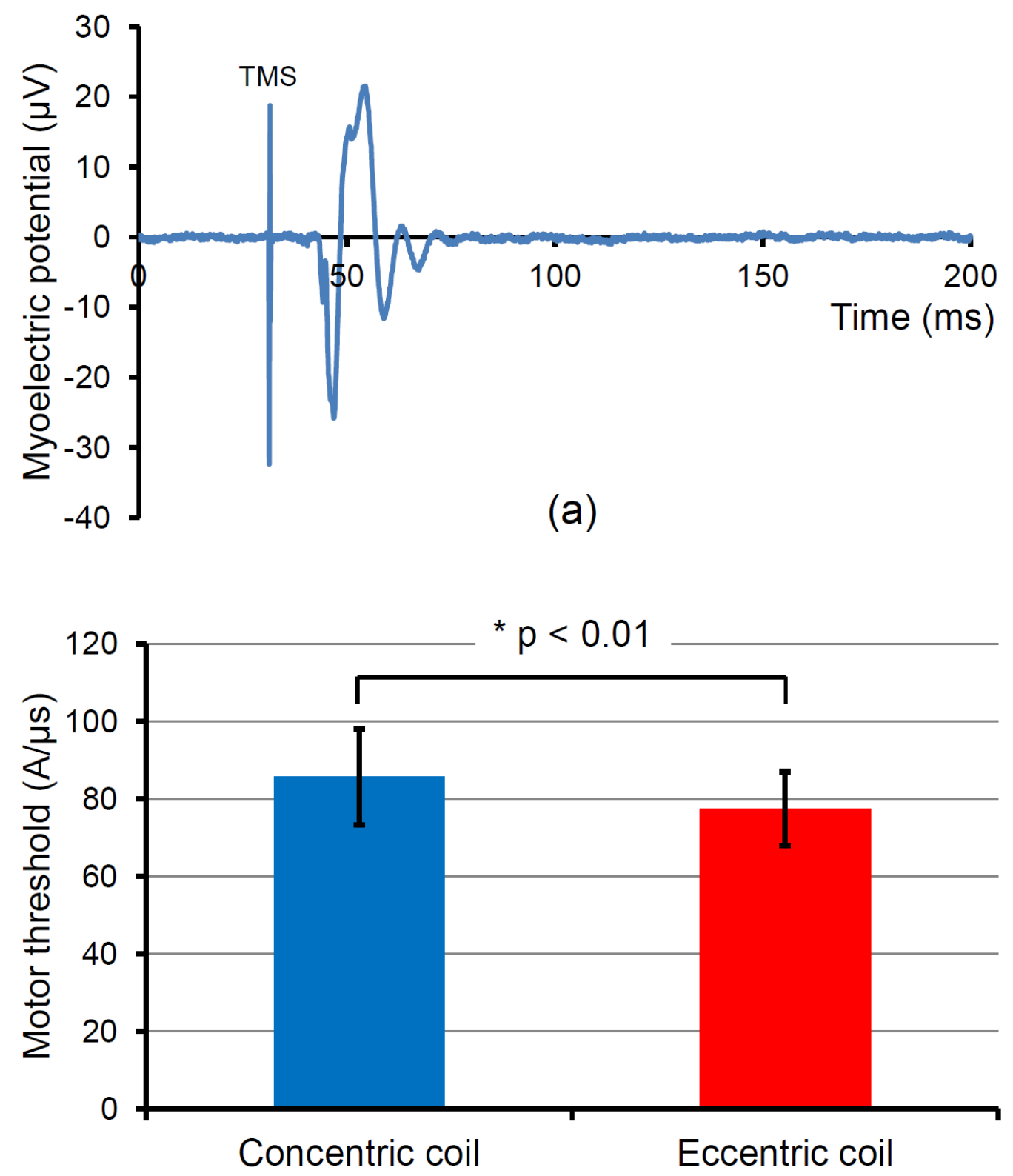

(b) 